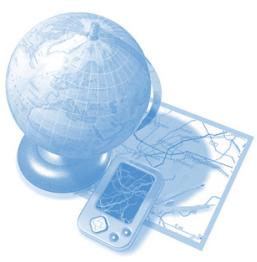

Derek Raine

TCETL

Department of Physics and

Astronomy

University of Leicester

Leicester LE1 7RH

jdr@le.ac.uk

\title{
From Projects to Problems
}

\begin{abstract}
Projects are a familiar feature of physics curricula and many courses include one or more group projects as a way of developing group work skills, if not for teaching physics. Problem-based learning on the other hand, which is designed primarily to teach physics while enhancing group work skills, is not so familiar. In this article we shall show how project work can be developed rather simply into problem-based learning by contextualising the project in terms of a problem and a viewpoint. The examples given will be based on developments of first and second year courses at Leicester to integrate practical, computational and theoretical work within the programme of specialist options. The benefits to staff and students will be discussed.

\section{Introduction}

Final year projects are now such an established part of the undergraduate curriculum that it is difficult to appreciate that when I started as a lecturer in physics they were, as I recall, a recent innovation in a few pioneering Departments. Group projects on the other hand, while widespread, are not universal in physics curricula, and even when they are present they are often considered as peripheral. Indeed, their justification is often expressed in terms of the development of group skills and team work rather than the teaching of physics content. This paper describes the history of our recent development of group projects at Leicester to support teaching of specialised options. A particular feature of these projects is the integration of practical and theory. The result might be described as the introduction of problem-based learning through the back door, as it were. The comparison with the core component of problem-based learning (the approach through the front door so to speak) has been instructive.
\end{abstract}

\section{Background}

In problem-based learning (PBL) students start from a problem or puzzle and, working in groups, seek out the new knowledge they need to solve the problem. A good PBL problem or puzzle is usually described as 'real world', but the essential point is that it should be expressed in such a way as to engage students, which often, but not always, means that it should relate to 'real life' (in this context meaning as experienced by physicists in their work). $\ln ^{1}$ Savin-Baden offers a number of distinctions between PBL problems and projects, not all entirely convincing. The main difference for our purposes is that projects are used to introduce open-ended research in a novel context, probably not duplicated from year to year and probably not conducted as a group exercise. PBL on the other hand, as it is usually implemented, has specific content objectives within the agreed curriculum.

The distinction is not a sharp one. Our own 'rocket project' for example illustrates to some extent the overlap. There is a given problem, in this case to build a rocket to launch a payload that will obtain scientific data which can be recovered and analysed. (A simple example might be to measure the vertical variation of the Earth's magnetic field at the launch site.) The students work in pairs on different aspects of the problem (design, electronics, data analysis and so on), so this is not PBL; on the other hand a subset at least of the core learning outcomes are common from year to year, the investigation is not essentially novel, and the students have to work as a group to fit the different aspects together, so this is not a standard final year project and bears many of the hallmarks of PBL. 
A key feature of the rocket project, shared by good PBL problems and final year projects, is that it generates a lot of student enthusiasm and engagement. The question then arises as to whether the lessons of the rocket project can be applied to core learning. For our immediate purpose we identify the 'core' as the compulsory components of our various specialist degree streams (we call them physics 'flavours') of physics with astrophysics, space science, nanoscience, planetary science and plain 'vanilla' physics.

\section{Why change?}

Change is expensive, and although some new lecturers may get a lighter teaching load to set up their courses, beyond that it is never entirely clear where the resources for teaching developments are supposed to come from. Thus, change needs a strong motivation and the promise that it will lead to greater efficiency once implemented (usually meaning less staff resources rather than better student results, but at least consistent with not significantly enlarged resources and no less a student experience). So what were the motivators?

1. The effectiveness of the standard laboratory in fostering the development of physical understanding has often been questioned and various alterations have been proposed to address this (eg Johnstone et $\mathrm{al}^{3}$ ). For example, our students who have followed the laboratory script to construct their own numerical stellar models, still find difficulty in answering examination problems on stellar structure.

2. Conversely, one might query the effectiveness of lectures in conveying information about practical aspects of a subject.

3. Practical work, whatever the benefits of the previous regime, had become a chore for staff and not very exciting for most (not all!) students. The laboratory experiments were tired and needed renewal, but there was little enthusiasm for doing this.

In introducing elements of PBL to combine some of the laboratory work with the presentation of theory, we had in mind that it would enable students to experience being, say, an astrophysicist (at some level), not just learning about astrophysics, in much the same way that our rocket project enables students to experience being a rocket scientist, not just to hear about how it is done.

\section{Some example projects}

To give a flavour of the projects I will outline a few examples (The intention is that further details should be available on the web in due course through the $\pi C E T L$ and the project LeAP problem bank, www.le.ac.uk/leap).

\section{Observational astronomy, year 1}

This project involves the construction of a telescope, by students working in pairs, from some supplied lenses, a digital camera, computer and software and a cardboard tube which can be cut to a length of their choice. Students must characterise the optical properties of their telescope (resolving power, chromatic aberration, calibration using standard simulated stars and so on), which cannot be obtained by asking the laboratory demonstrators (or their colleagues) since the configuration is unique to the particular pair of students. Students then observe a simulated variable star system. This is a binary system with a period of 6 hours, much longer than the length of the laboratory session. A number of pairs of students (from different sessions) must then exchange information to determine the properties of the binary system. They can only do this effectively if the calibrations of their telescopes are correct. Thus, there is no prior known right answer to this, but students have to get the answer right to solve the problem. (And, incidentally, understand the sources of error and evaluate them correctly!)

2. Nanoparticles, year 1 This project involves a novel way of making nanoparticles of sodium chloride by thermal evaporation of a spray of salt solution. Students have to set up the experiment, including a calculation of the appropriate concentration of solute, and attempt to characterise the particles by optical absorption.

They also get to look at the nanoparticles in an atomic microscope to confirm their estimates.

3. Stereo-Imager, year 2

Students are asked to design, build and test a (small scale) stereoscopic imaging device for a planetary rover that can be used to estimate distances and to project green/red stereo images.

Each of the projects requires students to seek out the relevant theory and put it into practice. Some of the projects integrate an element of computation also. 


\section{Development}

The projects were developed largely by recent graduates working over the summer period under the supervision of the responsible academic member of staff with a variable degree of interference from myself, mainly to try to guide the projects away from a reinvention of the standard scripted laboratory practical and towards a problem to be solved with the necessary support materials (and to sign the requests for just another few (hundred) pounds of equipment).

\section{Structure}

Both years are run in the same way. Each laboratory session (4 in year 1, 6 in year 2) is preceded by a group tutorial session with the member of staff in charge in which progress is presented and discussed and objectives for the next laboratory session are agreed, including the relevant preliminary research. (Obviously the first and last sessions are slightly different.) Staff can provide whatever information they think is relevant, but they are asked not to turn the tutorial into a lecture. Staff supervise the laboratory work to a variable degree, but in all cases are present for only a part of a session.

While there is clearly a close relationship with standard group projects there are also some differences. The presentation of the projects to students is couched in the language of a problem rather than an endpoint. So for example, the deliverable in the stereo-imager project is not the hardware, but the solution to the problem of determining distances. There is a focus on process as well as endpoint. By this I mean that the group is expected to operate within a defined structure, not just left to get on with it. We use our local PBL model for this ${ }^{4}$, although any PBL structure would be equally applicable. The performance of the group process is assessed (rather lightly), not just the deliverables.

\section{Some outcomes}

We do not yet have the results of a formal evaluation process from interviews that have been carried out independently with staff and students or from student questionnaires. However, what we do have is intriguingly mixed. Anecdotally, and from casual observation, there was a much greater engagement of staff with their projects than has been common in the laboratory with standard experiments. The student viewpoint is difficult to disentangle, partly because they have not experienced the experiments and lecture courses that have been replaced.
There appears generally to be a greater involvement (despite one comment 'why can't we just do ordinary labs?'), but as yet no evidence of greater retention of subject knowledge.

Further research will hopefully tell us what more we can learn about embedding PBL in the physics core. One preliminary observation, which comes across strongly, is that students pick up very quickly on the degree of staff engagement; it may be this aspect (and the now well-known positive effect of collaborative learning ${ }^{5}$ ) that dominates the responses to PBL.

\section{References}

1. Savin-Baden, M., Facilitating Problem-based Learning , McGraw Hill (2003)

2. http://www.star.le.ac.uk/rockets/project.shtml

3. Johnstone, A. H., Watt, A. and Zamen, T. U., The Students' Attitude and Cognition Change to a Physics Laboratory, Physics Education, 35(1), 22 - 28 (1998) 4. Raine, D. J. and Symons, S., PossiBiLities - a practice guide to $P B L$, Higher Education Academy Physical Sciences Subject Centre (2005)

5. Smith, et al., Pedagogies of Engagement : Classroom Based Practices, J. Eng. Ed. (2005) 